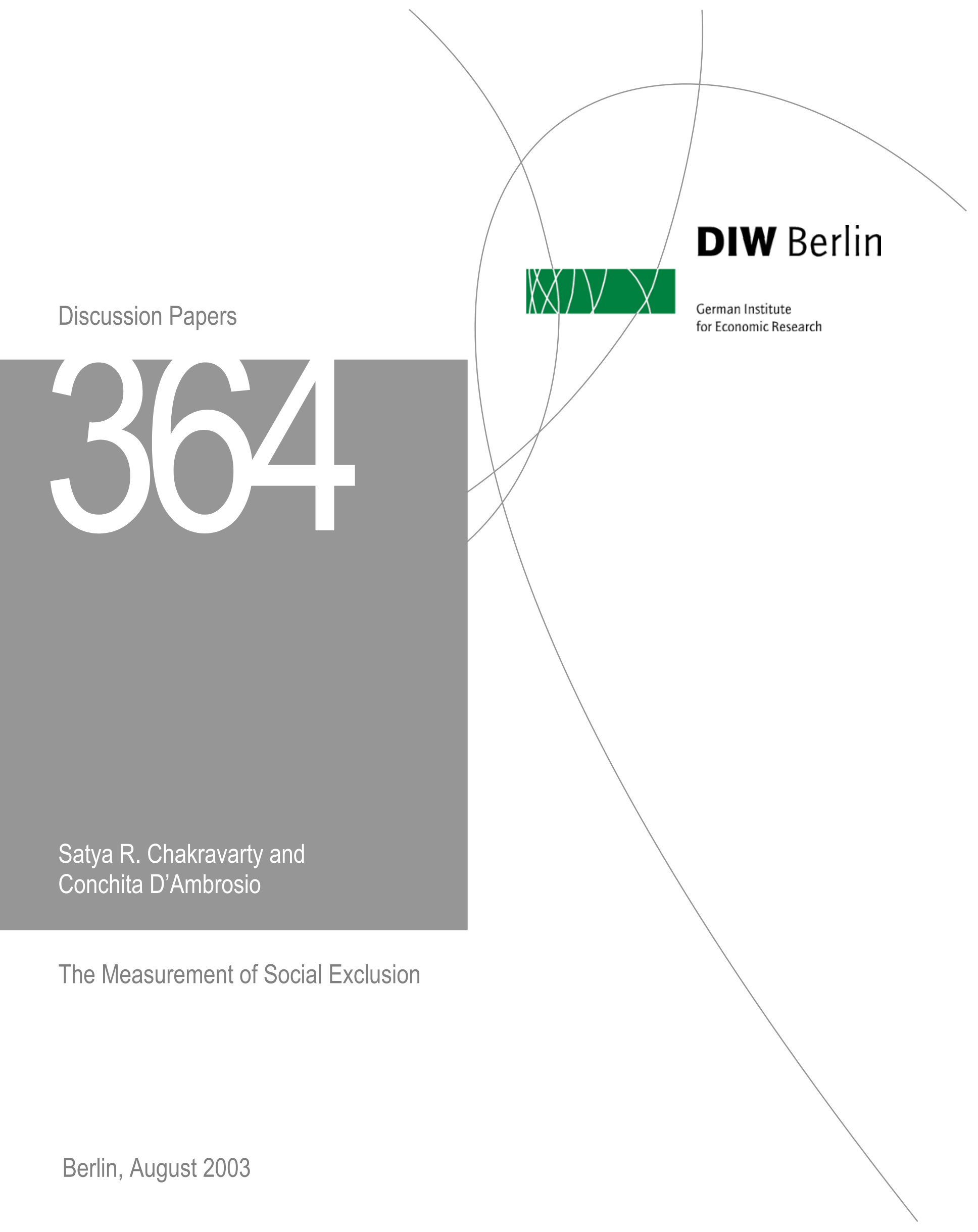


Opinions expressed in this paper are those of the author and do not necessarily reflect views of the Institute.

DIW Berlin

German Institute

for Economic Research

Königin-Luise-Str. 5

14195 Berlin,

Germany

Phone +49-30-897 89-0

Fax $\quad+49-30-89789-200$

www.diw.de

ISSN 1619-4535 


\title{
The Measurement of Social Exclusion*
}

\author{
Satya R. Chakravarty \\ Indian Statistical Institute, Calcutta \\ Conchita D'Ambrosio \\ Università Bocconi and DIW Berlin
}

(This version July 2003)

\begin{abstract}
This paper develops an axiomatic approach to the measurement of social exclusion. At the individual level, social exclusion is viewed in terms of deprivation of the person concerned with respect to different functionings in the society. At the aggregate level we treat social exclusion as a function of individual exclusions. The class of subgroup decomposable social exclusion measures using a set of independent axioms is identified. We then look at the problem of ranking exclusion profiles by exclusion dominance principle under certain restrictions. Finally, applications of decomposable and non-decomposable measures suggested in the paper using European Union and Italian data are also considered.
\end{abstract}

JEL Classification Number: D63.

Keywords: Social Exclusion, Axioms, Characterization, Dominance, Application, Policy.

\footnotetext{
${ }^{*}$ Corresponding author: Conchita D'Ambrosio. e-mail: cdambrosio@diw.de. Address: German Socio-Economic Panel Study (SOEP), DIW Berlin, Koenigin Luise Strasse 5, 14191 Berlin, Germany. Chakravarty wishes to thank Università Bocconi, Milano, for financial support and hospitality. D'Ambrosio acknowledges financial support from Università Bocconi, ricerca di base "Social Exclusion and Social Distance". We would like to thank Joan Esteban, James Foster, Patrick Moyes, Debraj Ray, Tony Shorrocks, Jacques Silber, Panos Tsakloglou, Claudio Zoli and the participants at the "Conflict and Polarization" meeting 2002, at the 2nd Journées d'Economie Publique LAGV, at the WIDER conference "Inequality, Poverty and Human Well-being", and at the ESPE 17th annual meeting for useful comments and suggestions.
} 


\section{Introduction}

The subject of this paper is the measurement of social exclusion. The broad questions that we try to address in this paper are: (i) When do we say that an individual is socially excluded; (ii) What is the level of social exclusion in a country? (iii) Can we say that social exclusion in country A is less than that in country B? (iv) Given the level of social exclusion in a society, which subgroups of the population, partitioned according to ethnic, geographic, or any other socioeconomic characteristic, contribute more to aggregate social exclusion? (v) When can we say that one society dominates another with respect to social exclusion and what are the consequences of such a dominance relationship?

Broadly speaking, a person is said to be socially excluded if she/he is unable to 'participate in the basic economic and social activities of the society in which she/he lives'. In the European Commission's Programme specification for 'targeted socioeconomic research', social exclusion is described as 'disintegration and fragmentation of social relation and hence a loss of social cohesion. For individual in particular groups, social exclusion represents a progressive process of marginalization leading to economic deprivation and various forms of social and cultural disadvantage'.

As Atkinson (1998) said, social exclusion is not just a consequence of unemployment. It is true that an unemployed person may not have income to maintain a subsistence standard of living and hence becomes socially excluded. But many employed persons may not be satisfied with their work or main activity. Expansion of employment may increase the income gap between low-paid and high-paid workers and hence it may not reduce or end social exclusion. Social exclusion may arise from the operations of the market and supplies of key goods and services. For instance, people may not be able to participate in the customary consumption activities because profit maximizing prices may exclude them from the markets. A person may not be allowed to have an account in a bank if he does not fulfil certain constraints. It can as well generate from operations of the State if the State's social security benefit programmes are targeted towards some particular groups or persons.

As social exclusion includes economic, social and political aspects of life, it is a multidimensional phenomenon. It implies deprivation in a wide range of indicators or functionings of living standards, which can be of quantitative or qualitative type. ${ }^{1}$

\footnotetext{
${ }^{1}$ See Atkinson, Cantillon, Marlier and Nolan (2002) for a list of functionings of social
} 
Social exclusion is related to both inequality and poverty, but should not be equated with either of them (Atkinson, 1998). According to Sen (1998), social exclusion is wider than poverty. Multidimensional inequality is a measure of the dispersion of the multidimensional distribution of quantities of consumption of the functionings for different individuals (Tsui, 1999). Multidimensional poverty measurement specifies a poverty threshold for each functioning, looks at the shortfalls of the functioning quantities of different individuals from the threshold levels, and aggregates these shortfalls into an overall magnitude of poverty (Bourguignon and Chakravarty, 2002). Thus, both multidimensional poverty and social exclusion deal with functionings failures, while in the former we view it in terms of the shortfalls from thresholds, in the latter the problem is one of inability to participate. Note further that in the case of both multidimensional inequality and poverty the functionings have to be of quantitative type, whereas social exclusion considers qualitative type functionings as well. Social exclusion can be regarded as a state and as a process leading to deprivation.

Atkinson (1998) argued further that it is a relative concept, we cannot say whether a person is socially excluded or not by looking at his position alone. The positions of the others in the society have to be taken into account for a proper implementation of any criterion for exclusion. It has, furthermore, a dynamic character because an individual is socially excluded if his deprivation continues or worsens over time.

Three types of implicit conceptualization of social exclusion are currently available in the literature. In the first, it is interpreted as the lack of participation in social institutions (Duffy, 1995, Rowntree Foundation, 1998, UK House of Commons, 1999, Paugam and Russell, 2000); whereas the second regards the problem as the denial or non-realization of rights of citizenship (Room, 1995, Klasen 1998). Finally, the third views social exclusion in terms of increase in distance among population groups (Akerlof, 1997). Some researchers attempted to suggest measures of social exclusion building on these approaches (see Bradshaw et al. 2000, Tsakloglou and Papadopoulos, 2001). However the theoretical foundations of these measures are often unclear.

In this paper we adopt an axiomatic approach to the measurement of social exclusion. ${ }^{2}$ Since in order to be socially integrated a person needs to have access to some social functionings, we first look at the functioning failure, that is, the number of functionings from which the person is excluded.

exclusion.

${ }^{2}$ An alternative approach has been proposed by Bossert et al. (2003). 
This number may be regarded as the deprivation score of the person under consideration. However, some of the functionings may be more important than others. Therefore, a more general way is to assign an integer weight to each failure depending on the importance of the functioning and the deprivation score of a person is the sum of these integers.

The social exclusion measure that we propose is a real valued function of the deprivation scores of different individuals in the society. In a sense our approach is similar to the view that considers social exclusion as lack of participation in social institutions, where lack of participation is treated in terms of functioning failures. We first characterize the family of exclusion measures whose members satisfy normalization, monotonicity, subgroup decomposability, and have nondecreasing marginals.

Normalization means that social exclusion is zero if nobody is socially excluded. Monotonicity requires the measure to increase if the deprivation score of a person increases.

According to subgroup decomposability, for any partitioning of the population with respect to some socioeconomic or demographic characteristic, the overall social exclusion is the population share weighted average of subgroup exclusion levels. This property enables us to calculate a particular subgroup's contribution to aggregate exclusion and hence to identify the subgroups that are more afflicted by exclusion and to implement anti-exclusion policy. Clearly, according to this notion of policy recommendation, an assessment of overall exclusion becomes contingent on the implicit valuation of the exclusion measure. However, an exercise of this type may be useful for two reasons. First, following Sen (1985), the non-welferist approach to policy analysis is becoming quite popular. Second, in many situations policy is evaluated using specific forms of measures. So it seems worthwhile to see what type of policy would be implied by the use of a specific exclusion measure.

Marginal social exclusion is defined as the change in social exclusion when we increase the deprivation score of a person by one. Nondecreasingness of marginal social exclusion ensures that in aggregating individual deprivation scores into an overall indicator of exclusion, a higher deprivation score does not get a lower weight than a lower score.

The characterized family of measures is shown to possess some additional interesting properties. It is also shown that the properties employed in the characterization exercise are independent, that is, none of these properties implies or is implied by another.

In order to fulfil subgroup decomposability the weights attached to different functionings should be independent of the population size. However, 
an alternative assumption, which appears to be quite realistic, is dependence of weights on the population size. It may also be worthwhile to study non-subgroup decomposable measures. We therefore consider two measures of this type, the symmetric mean exclusion of order $\nu>1$ and the Gini exclusion measure, and use population size dependent weights to calculate them.

Next, we consider the problem of ranking two societies by the social exclusion dominance criterion. We demonstrate that for two societies with a common population size and the same total deprivation score, if one dominates the other by the exclusion dominance criterion, then the former becomes at least as socially excluded as the latter by all additive social exclusion measures that satisfy anonymity and have nondecreasing marginals. This result parallels the if part of the well-known Atkinson (1970) result on Lorenz Domination which says that if $u$ and $v$ are two income distributions of a given total over a fixed population size, and if $u$ Lorenz dominates $v$, then all symmetric utilitarian social welfare functions regard $u$ at least as good as $v$, where the identical individual utility function is concave.

Finally, we apply different measures to the EU member states in the 1990's and consider some policy implications.

The paper is organized as follows. The next section introduces the formal framework for measuring social exclusion and presents the properties for an exclusion measure. In Section 3 we characterize the family of exclusion measures and discuss its properties. Section 4 deals with social exclusion dominance relation. The application is contained in Section 5. Section 6 concludes.

\section{Properties for a measure of social exclusion}

Let $\mathbf{N}\left(\mathbf{N}_{0}\right)$ be the set of all positive (nonnegative) integers and $\mathbf{R}$ be the set of real numbers. For all $n \in \mathbf{N}, D^{n}$ is the $n$-fold Cartesian product of $\mathbf{N}_{0}$ and $1^{n}$ is the $n$-coordinated vector of ones. For any society with a population of size $n \in \mathbf{N}$, there is a finite nonempty set of functionings $F$ relevant for social integration. Throughout this paper we assume that $F$ is fixed so that cross population comparisons of social exclusion can be made in terms of elements of $F{ }^{3}$ An individual in an $n$-person society can be excluded from any subset of $F$, where $n \in \mathbf{N}$ is arbitrary. The degree of exclusion or deprivation of a person can be captured using the number of functionings from which she/he is excluded. For each functioning, we define a characteristic function which

\footnotetext{
${ }^{3}$ See Atkinson et al. (2002) for commonness of elements of $F$ for the EU as a whole.
} 
takes on the value 1 or 0 according as the person is excluded or not from the functioning. Since some functionings may be more important than others, the characteristic function of each functioning is weighted by an integer, where the integer weights are determined in terms of importance of the functionings. ${ }^{4}$ The deprivation score of the person concerned is then given by the sum of integer weighted characteristic functions. More precisely, let $F_{i} \subseteq F$ be the set of functionings from which person $i$ is excluded. Denote the weight attached to attribute $j$ by $w_{j}$, then $x_{i}=\sum_{j \in F_{i}} w_{j}$.

This procedure of calculating the individual deprivation scores is quite similar to the Basu-Foster (1998) way of determining a household literacy profile. They assumed that individual literacy is a $0-1$ variable and an adult member of a household is identified by the number 0 or 1 according as he is illiterate or literate. The total number of literates in the household is then simply the sum of the 1's in the household. This procedure can as well be extended to the situation when literacy is assumed to be multidimensional.

An exclusion profile in a society of $n$ person is a vector $x=\left(x_{1}, \ldots, x_{n}\right)$, where $x_{i} \in \mathbf{N}_{0}$ is the deprivation score of person $i$. We assume that the calculation of $x_{i}$ involves a dynamic or longitudinal aspect. ${ }^{5}$ If $x_{i}$ is positive, trade-off between excluded and non-excluded functionings is not allowed. For instance, a person's high income cannot compensate the dissatisfaction associated with his job. The set of exclusion profiles for an $n$-person population is $D^{n}, n \geq 1$. Thus, $x \in D^{n}$ for some $n \in \mathbf{N}$. The set of all possible exclusion profiles is $D=\bigcup_{n \in \mathbf{N}} D^{n}$. A measure of social exclusion is a function $E: D \rightarrow \mathbf{R}$. For any $n \in \mathbf{N}$, the restriction of $E$ on $D^{n}$ is given by $E^{n}$. For any $n \in \mathbf{N}, x \in D^{n}, E^{n}(x)$ is a measure of the extent to which different individuals are excluded from the activities taking place in the society, that is, the degree of exclusion suffered by all individuals in the society as a whole. For all $n \in \mathbf{N}, x \in D^{n}$, let $S(x)$ be the set of persons with positive deprivation scores, that is $S(x)=\left\{i, 1 \leq i \leq n \mid x_{i}>0\right\}$. For any $n \in \mathbf{N}, x \in$ $D^{n}$, let $q$ be the cardinality of $S(x)$, that is the number of persons in $S(x)$. For any $n \in \mathbf{N}, x \in D^{n}$, we write $\bar{x}$ for nonincreasingly ordered permutation of $x$, that is $\bar{x}_{1} \geq \bar{x}_{2} \geq \ldots \geq \bar{x}_{n}$.

We assume that an arbitrary exclusion measure $E: D \rightarrow \mathbf{R}$ should satisfy the following postulates.

Axiom 1 : Normalization (NOM): For all $n \in \mathbf{N}, E^{n}\left(01^{n}\right)=0$.

Axiom 2 : Monotonicity (MON): For any $n \in \mathbf{N}, x \in D^{n}$ and for any $i$,

\footnotetext{
${ }^{4}$ See Section 5 for one approach to the calculation of weights.

${ }^{5}$ See Section 5 for details.
} 
$1 \leq i \leq n$

$$
E^{n}(x)<E^{n}\left(x_{1}, \ldots, x_{i-1}, x_{i}+c, x_{i+1}, \ldots, x_{n}\right),
$$

where $c \in \mathbf{N}$.

Axiom 3 : Nondecreasingness of Marginal Social Exclusion (NMS): For any $n \in N, x \in D^{n}$, and for any $i, j, 1 \leq i, j \leq n$, if $x_{i} \geq x_{j}$ then:

$$
\begin{gathered}
E^{n}\left(x_{1}, \ldots, x_{i-1}, x_{i}+1, x_{i+1}, \ldots, x_{j-1}, x_{j}, x_{j+1}, \ldots, x_{n}\right)-E^{n}(x) \geq \\
E^{n}\left(x_{1}, \ldots, x_{i-1}, x_{i}, x_{i+1}, \ldots, x_{j-1}, x_{j}+1, x_{j+1}, \ldots, x_{n}\right)-E^{n}(x) .
\end{gathered}
$$

Axiom 4 : Subgroup Decomposability (SUD): For any $x^{i} \in D^{n_{i}}, i=1, \ldots, k$, $E^{n}(x)=\sum_{i=1}^{k} \frac{n_{i}}{n} E^{n_{i}}\left(x^{i}\right)$, where $x=\left(x^{1}, x^{2}, \ldots, x^{k}\right)$.

Axiom 5 : Population Principle (POP): For all $n \in \mathbf{N}, x \in D^{n}, E^{m n}(y)=$ $E^{n}(x)$, where $y=\left(x^{1}, x^{2}, \ldots, x^{m}\right), m \geq 1$ and each $x^{i}=x$.

Axiom 6 : Anonymity (ANY): For all $n \in \mathbf{N}, x \in D^{n}, E^{n}(x)=E^{n}(x P)$, where $P$ is any $n \times n$ permutation matrix. ${ }^{6}$

Normalization is a miniminality principle. It says that if nobody is excluded from any functioning in the society, then the value of the social exclusion measure is zero. Monotonicity says that if the deprivation score of an individual increases, then social exclusion should increase. If a social exclusion measure satisfies $N O M$ and $M O N$, then it will take a positive value if at least one individual has a positive deprivation score.

Sen (1976) argued that in income poverty measurement the poverty line can be taken as the reference point for all poor persons and the poverty gap of a poor, his income shortfall from the poverty line, is a measure of deprivation suffered by him. In order to attach higher weight to higher deprivation, Sen assumed that the weight on individual $i$ 's poverty gap is equal to his rank in the income distribution of the poor. This guarantees that an increase in poverty due to a reduction in the income (increase in deprivation) of a poor will be higher the lower (higher) is the income (deprivation) of the poor. Conversely, in order that an increase in poverty due to reduction in the income of a poor is higher the lower the income of the poor is, a necessary condition is to attach higher weight lower down the income scale. Our NMS postulate has a similar spirit. We consider two persons where the deprivation score of the first is not lower than that of the second. Then the change in

\footnotetext{
${ }^{6} \mathrm{An} n \times n$ matrix is a permutation matrix if each of its entries is either zero or one, and each of its rows and columns sums to one.
} 
social exclusion, if the deprivation score of the former increases by one, is at least as large as the corresponding change when the deprivation score of the latter increases by the same amount. It is clear that the postulates NOM and $N M S$ capture the idea that exclusion is a relative phenomenon.

$S U D$, which expresses aggregate exclusion in a society as a weighted average of subgroup exclusion levels, where the weights are population shares of the subgroups, is very important from policy point of view. $\frac{n_{i}}{n} E^{n_{i}}\left(x^{i}\right)$ is the contribution of subgroup $i$ to total exclusion, i.e., the amount by which social exclusion will decrease if exclusion in subgroup $i$ is eliminated. $\left(\frac{n_{i} E^{n_{i}}\left(x^{i}\right)}{n E^{n}(x)}\right) 100$ is the percentage contribution of subgroup $i$ to total exclusion. Each of these figures is useful to planners and analysts to formulate anti-exclusion policies. It may be important to note that if $x_{i}$ 's are dependent on the population size, $S U D$ may be violated.

$P O P$ says that if an exclusion profile is replicated several times, then the social exclusion of the original and the replicated profiles are the same. Clearly, POP leads us to view exclusion in average terms. It is helpful for cross population comparisons of exclusions.

Finally, $A N Y$ means that the exclusion measure is symmetric, i.e. any reordering of the deprivation scores leaves the exclusion level unchanged. $A N Y$ is unavoidable as long as the individuals are not distinguished by anything other than deprivation scores.

\section{The family of subgroup decomposable social ex- clusion measures}

In this section we derive the class of social exclusion measures whose members satisfy $N O M, M O N, N M S$, in addition to $S U D$. Let $\Phi$ be the class of all functions $f: \mathbf{N}_{0} \rightarrow \mathbf{R}$ such that $f(0)=0, f$ is increasing, and $f$ has a nondecreasing marginal, that is:

$$
f\left(x_{i}+1\right)-f\left(x_{i}\right) \geq f\left(x_{j}+1\right)-f\left(x_{j}\right),
$$

where $x_{i} \geq x_{j}$.

For theorems 1 and 2 of this section we assume that the weights attached to different functionings are independent of the population size.

We then have:

Theorem 1 : A social exclusion measure $E: D \rightarrow \mathbf{R}$ satisfies $N O M, M O N$, 
$N M S$, and SUD if and only if for all $n \in \mathbf{N}, x \in D^{n}$,

$$
E^{n}(x)=\frac{1}{n} \sum_{i \in S(x)} f\left(x_{i}\right),
$$

where $f$ is a member of $\Phi$.

\section{Proof:}

Let $n \in \mathbf{N}$ and $x \in D^{n}$ be arbitrary. Then by repeated applications of SUD:

$$
E^{n}(x)=\frac{1}{n} \sum_{i=1}^{n} E^{1}\left(x_{i}\right)
$$

We can rewrite $E^{n}$ in (3) as:

$$
E^{n}(x)=\frac{1}{n} \sum_{i=1}^{n} f\left(x_{i}\right),
$$

where $f=E^{1}$. Clearly, $f: \mathbf{N}_{0} \rightarrow \mathbf{R}$. MON demands increasingness of $f$. Now, suppose $x_{i} \geq x_{j}$. The inequality:

$$
\begin{gathered}
E^{n}\left(x_{1}, \ldots, x_{i-1}, x_{i}+1, x_{i+1}, \ldots, x_{j-1}, x_{j}, x_{j+1}, \ldots, x_{n}\right)-E^{n}(x) \geq \\
E^{n}\left(x_{1}, \ldots, x_{i-1}, x_{i}, x_{i+1}, \ldots, x_{j-1}, x_{j}+1, x_{j+1}, \ldots, x_{n}\right)-E^{n}(x),
\end{gathered}
$$

on simplification, reduces to:

$$
\begin{aligned}
& f\left(x_{i}+1\right)-f\left(x_{i}\right) \geq \\
& f\left(x_{j}+1\right)-f\left(x_{j}\right),
\end{aligned}
$$

which is nondecreasingness of marginal of $f$. Clearly, if $x_{i}=0$ for all $i$, then $N O M$ requires that $f(0)=0$.

Obviously, $f(0)=0$ enables us to rewrite $\frac{1}{n} \sum_{i=1}^{n} f\left(x_{i}\right)$ as $\frac{1}{n} \sum_{i \in S(x)} f\left(x_{i}\right)$. This establishes the necessity part of the theorem on $D^{n}$ for a given $n \in \mathbf{N}$.

The sufficiency is easy to verify. Since $n \in \mathbf{N}$ was chosen arbitrarily, our result holds for all $n \in \mathbf{N}$.

Note that the general measure in (2) satisfies $A N Y$ and $P O P$ although we did not use these properties in its derivation. We can interpret $f$ in (2) as the individual exclusion function. An alternative way of writing the formula (2) is:

$$
E^{n}(x)=\frac{H}{q} \sum_{i \in S(x)} f\left(x_{i}\right)
$$


where $H=\frac{q}{n}$ is the head-count measure of social exclusion, the proportion of persons that is socially excluded in the population. For a fixed $n$, on social exclusion profiles with a given $q, H$ is a constant function. Thus $H$ is violator of $M O N$ although it meets $N O M, S U D, P O P, A N Y$, and $N M S$.

The head-count measure of social exclusion is quite analogous to the multidimensional poverty head-count ratio. Multidimensional poverty measurement considers for each person a poverty indicator variable that takes on the value of 1 if his consumption of some attribute(s) falls below the corresponding threshold(s). Otherwise the indicator variable assumes the value zero. The total number of multidimensional poor is then given by the sum of indicator variables across persons (see Bourguignon and Chakravarty, 2003).

In order to illustrate the general formula in (2), let $f \in \Phi$ be of the form $f(t)=t^{\delta}, \delta \geq 1$. Then the corresponding measure is:

$$
E_{\delta}^{n}(x)=\frac{H}{q} \sum_{i \in S(x)} x_{i}^{\delta} .
$$

For any $\delta \geq 1, E_{\delta}^{n}$ satisfies all the postulates. For $0<\delta<1, E_{\delta}^{n}$ is a violator of $N M S$ but not of others. As $\delta \rightarrow 0, E_{\delta}^{n} \rightarrow H$. The single parameter $\delta$ in (6) is a value judgement parameter. $E_{\delta}^{n}$ becomes more sensitive to the higher deprivation scores as $\delta$ increases from 2 to plus infinity. For a given $x \in D^{n}$, an increase in the value of $\delta$ does not decrease $E_{\delta}^{n}$. For $\delta=1, E_{\delta}^{n}$ becomes the average deprivation score of the society, that is, $A=\frac{1}{n} \sum_{i \in S(x)} x_{i}$. For $\delta=2$, we can rewrite $E_{\delta}^{n}$ as:

$$
E_{\delta}^{n}(x)=\sigma^{2}(x)+A^{2}(x),
$$

where $\sigma^{2}$ is the variance of the society deprivation scores. Given $A$, a reduction in $\sigma^{2}$ reduces the measure in (7). Such a situation may arise if a higher deprivation score decreases and a lower deprivation score increases by the same amount. Over social exclusion profiles with the same population size and the same average deprivation score, the ranking of the profiles generated by $E_{\delta}^{n}($ for $\delta=2)$ is the same as that generated by $\sigma^{2}$.

An alternative of interest arises from the specification $f(t)=e^{\alpha t}-1$, where $\alpha>0$. The resulting measure is:

$$
E_{\alpha}^{n}(x)=\frac{H}{q} \sum_{i \in S(x)}\left(e^{\alpha x_{i}}-1\right) .
$$


For a given $x \in D^{n}, E_{\alpha}^{n}$ is nondecreasing in $\alpha . E_{\alpha}^{n}$ satisfies all the properties for all positive $\alpha$. As $\alpha$ increases, the underlying evaluation attaches more weight to the higher deprivation scores.

We will now show that the postulates $N O M, M O N, N M S$ and $S U D$ are independent. By independence we mean that if one of these postulates is dropped, then there will be measures that will satisfy the remaining postulates, but not the dropped one.

Theorem 2 : The properties NOM, MON, NMS, and SUD are independent.

\section{Proof:}

(a) Evidently the measure $I_{1}^{n}(x)=\frac{1}{n} \sum_{i \in S(x)} e^{x_{i}}$ is not normalized, but it will fulfil the other properties.

(b) Since the measure $I_{2}^{n}(x)=-\frac{1}{n} \sum_{i \in S(x)} \frac{x_{i}}{1+x_{i}}$ is decreasing in $x_{i}$, it is a violator of $M O N$, but not of the remaining postulates.

(c) The measure $I_{3}^{n}(x)=\frac{1}{n} \sum_{i \in S(x)} x_{i}^{\theta}, 0<\theta<1$, has a decreasing marginal and hence it fails to satisfy $N M S$, but it verifies the other properties.

(d) Since the measures $I_{4}^{n}(x)=\left(\frac{1}{n} \sum_{i \in S(x)} x_{i}^{\nu}\right)^{\frac{1}{\nu}}, \nu>1$, and $I_{5}^{n}(x)=$ $\frac{1}{n^{2}} \sum_{i \in S(x)} \bar{x}_{i}(2(n-i)+1)$ are not additive across components, they are not subgroup decomposable. However, they are normalized, monotonic, and have increasing marginals.

The measure $I_{4}^{n}$ is the symmetric mean exclusion of order $\nu(>1)$. We can refer to $I_{5}^{n}$ as the Gini exclusion measure since it involves a Gini type averaging. ${ }^{7}$ Because of non-additivity we can calculate these two measures using both population size dependent and independent weights. Since subgroup decomposable measures require the latter type of weights, our calculation of these two measures in the next section use the former weighting scheme.

It is clear that to every individual exclusion function $f \in \Phi$, there corresponds a different social exclusion measure of the form (2). They will differ only in the manner how a person's individual exclusion is specified as a function of his deprivation score. However, there is no guarantee that these social exclusion measures will rank exclusion profiles in the same way. We consider the problem of ranking exclusion profiles in the next section.

\footnotetext{
${ }^{7}$ Strictly speaking, when incomes are arranged in non-increasing order, the Gini index of inequality can be written as a linear function with weights being the odd natural numbers in increasing order. Since the averaging in $I_{5}^{n}$ is quite similar in nature, we call it the Gini social exclusion measure.
} 


\section{The social exclusion dominance relation}

We begin this section by defining the social exclusion dominance criterion and look at its implications for exclusion profiles with a fixed total over a given population size.

For $x, y \in D^{n}$, we say that $x$ dominates $y$ by the social exclusion relation, which we write $x \succeq_{S E} y$, if:

$$
\sum_{j=1}^{k} \bar{x}_{j} \geq \sum_{j=1}^{k} \bar{y}_{j},
$$

for all $k=1,2, \ldots, n$.

Given that the exclusion profiles $\bar{x}$ and $\bar{y}$ are ranked in nonincreasing order of functioning failures of the individuals, $x \succeq_{S E} y$ demands that the cumulative deprivation score of the first $k$ persons in $\bar{x}$ is at least as large as that in $\bar{y}$, where $k=1,2, \ldots, n$.

In order to study implications of the relation $\succeq_{S E}$ in terms of exclusion measures, we now have the following:

Definition 1 : For any $x \in D^{n}$, we say that $\bar{y}$ is obtained from $\bar{x}$ by a favourable composite change (FCC) if:

$$
\begin{aligned}
& \bar{y}_{i}=\bar{x}_{i}-1 \\
& \bar{y}_{j}=\bar{x}_{j}+1 \\
& \bar{y}_{k}=\bar{x}_{k} \quad \text { for all } k \neq i, j,
\end{aligned}
$$

where $\bar{x}_{i}>\bar{x}_{j}$.

An FCC, which explicitly involves relativity issue of exclusion, means that the degree of exclusion of a more deprived person $(i)$ is reduced by 1 , whereas that of a less deprived person $(j)$ is increased by 1 , so that the total scores in the two profiles are the same, but the variance of the new profile $(\bar{y})$ is less than that of the original one $(\bar{x})$. Note that the transformation in (10) does not alter the relative positions of the affected individuals and it reduces the deprivation score of the worse off person $(i)$. This is the reason why we call it an FCC.

Marshall and Olkin (1979) defined a special kind of linear transformation, called a $T$-transformation, of a vector that leaves all but two components of the vector unchanged, and replaces these two components by averages. An $F C C$ is a $T$-transformation since:

$$
\begin{array}{ll}
\bar{y}_{i}=\lambda \bar{x}_{i}+(1-\lambda) \bar{x}_{j} & \\
\bar{y}_{j}=(1-\lambda) \bar{x}_{i}+\lambda \bar{x}_{j} & \\
\bar{y}_{k}=\bar{x}_{k} & \text { for all } k \neq i, j,
\end{array}
$$


where $\lambda=\frac{\left(\bar{x}_{i}-\bar{x}_{j}-1\right)}{\left(\bar{x}_{i}-\bar{x}_{j}\right)}$.

The following theorem gives an interesting consequence of the relation $\succeq_{S E}$ for additive exclusion measures that satisfy anonymity and have nondecreasing marginals.

Theorem 3 : Let $x, y \in D^{n}$, where $\sum_{l=1}^{n} x_{l}=\sum_{l=1}^{n} y_{l}$. Then $x \succeq_{S E} y$ implies that $\sum_{l=1}^{n} h\left(x_{l}\right) \geq \sum_{l=1}^{n} h\left(y_{l}\right)$ for all individual exclusion measures $h: \mathbf{N}_{0} \rightarrow \mathbf{R}$ whose marginals are nondecreasing.

\section{Proof:}

Muirhead (1903) showed that given $x, y \in D^{n}$ along with $\sum_{l=1}^{n} x_{l}=$ $\sum_{l=1}^{n} y_{l}$, if $x \succeq_{S E} y$ holds, then $\bar{y}$ can be derived form $\bar{x}$ by successive applications of a finite number of FCCs. Assume, without loss of generality, that only one $F C C$ affecting individuals $i$ and $j$, where $\bar{x}_{i}>\bar{x}_{j}$, takes us from $\bar{x}$ to $\bar{y}$.

Given $\bar{x}_{i}>\bar{x}_{j}$, let $\theta=\bar{x}_{i}-\bar{x}_{j}-1$. Note that $\theta \in \mathbf{N}_{0}$. Since the marginal of the individual exclusion function $h$ is nondecreasing, we have:

$$
h\left(\bar{x}_{j}+1\right)-h\left(\bar{x}_{j}\right) \leq h\left(\bar{x}_{j}+\theta+1\right)-h\left(\bar{x}_{j}+\theta\right),
$$

which we can rewrite as:

$$
h\left(\bar{x}_{j}+1\right)-h\left(\bar{x}_{j}\right) \leq h\left(\bar{x}_{i}\right)-h\left(\bar{x}_{i}-1\right) .
$$

Inequality (13) on rearrangement gives:

$$
h\left(\bar{x}_{j}+1\right)+h\left(\bar{x}_{i}-1\right) \leq h\left(\bar{x}_{i}\right)+h\left(\bar{x}_{j}\right) .
$$

Substituting the values of $\bar{x}_{j}+1$ and $\bar{x}_{i}-1$ in (14), we get:

$$
h\left(\bar{y}_{j}\right)+h\left(\bar{y}_{i}\right) \leq h\left(\bar{x}_{i}\right)+h\left(\bar{x}_{j}\right) .
$$

Inequality (15) along with the information that $\bar{y}_{k}=\bar{x}_{k}$ for all $k \neq i, j$ gives us:

$$
\sum_{l=1}^{n} h\left(\bar{y}_{l}\right) \leq \sum_{l=1}^{n} h\left(\bar{x}_{l}\right) .
$$

Since the social exclusion measure $\sum h($.$) satisfies anonymity, we can rewrite$ (16) as:

$$
\sum_{l=1}^{n} h\left(y_{l}\right) \leq \sum_{l=1}^{n} h\left(x_{l}\right),
$$


which is the desired result.

Theorem 3 is very valuable. It shows how an $F C C$ becomes helpful in ranking two exclusion profiles. It also provides a justification for using $N M S$ as a postulate for a social exclusion measure.

In an $F C C$ the deprivation scores of the two affected persons change in opposite directions. But often unidirectional changes in the scores of the two or more persons may take place. The following result, whose proof can be found in Fulkerson and Ryser (1962), states that under certain conditions the relation $x \succeq_{S E} y$, where the total scores in $x$ and $y$ are the same, is preserved.

Theorem $4:$ Let $x, y \in D^{n}$, where $\sum_{i=1}^{n} x_{i}=\sum_{i=1}^{n} y_{i}$, be arbitrary. Then $x \succeq_{S E} y$ implies that $\left(\bar{x}-e_{j}\right) \succeq_{S E}\left(\bar{y}-e_{i}\right)$, where $i \leq j$ and $e_{k}$ is the $n$-coordinated vector with 1 in the $k^{\text {th }}$ position and zeros elsewhere.

The intuitive appeal of Theorem 4 is quite clear. Given that $x$ dominates $y$ if we reduce the degree of exclusion of one person in $\bar{x}$ and one person in $\bar{y}$, where the latter is relatively worse off than the former, the exclusion dominance remains preserved.

The following result, whose formal proof can be found in Fulkerson and Ryser (1962), is a generalization of Theorem 4.

Theorem 5 : Let $x, y \in D^{n}$, where $\sum_{i=1}^{n} x_{i}=\sum_{i=1}^{n} y_{i}$, be arbitrary. Let $u$ be obtained from $\bar{x}$ by reducing deprivation scores of persons in positions $i_{1}, i_{2}, \ldots, i_{k}$ by 1 . Similarly, suppose $v$ is obtained from $\bar{y}$ by reducing deprivation scores of persons in positions $j_{1}, j_{2}, \ldots, j_{k}$. If $i_{1} \leq j_{1}, i_{2} \leq j_{2}, \ldots, i_{k} \leq$ $j_{k}$ and $x \succeq_{S E} y$, then $u \succeq_{S E} v$.

\section{An empirical illustration}

The purpose of this section is to illustrate the social exclusion measures $E_{\delta}$ in (6), $I_{4}$, the symmetric mean exclusion of order $\nu$, and $I_{5}$, the Gini exclusion measure using the European Community Household Panel (ECHP) data. ${ }^{8}$ Since $I_{4}$ and $I_{5}$ are calculated to illustrate non-subgroup decomposability, we calculate them here using population size dependent weights for different

\footnotetext{
${ }^{8}$ Since our illustration involves cross population comparisons, we drop the superscript $n$ from $E_{\delta}^{n}, I_{4}^{n}$, and $I_{5}^{n}$.
} 
functionings. We base our analysis on the first six waves of ECHP, which cover the period from 1994 to 1999. The surveys are conducted at a European national level. The ECHP is an ambitious effort at collecting information on the living standards of the households of the EU member-states using common definitions, information collection methods and editing procedures. It contains detailed information on incomes, socio-economic characteristics, housing amenities, consumer durables, social relations, employment conditions, health status, subjective evaluation of well-being, etc. Of the $15 \mathrm{EU}$ member-states, we could not consider Austria, Finland, Luxembourg, and Sweden since the data for these countries were not available for all the waves. For similar reasons we had to exclude Germany and the UK. In particular, the ECHP surveys of these countries were substituted by national surveys, SOEP and BHPS respectively, that did not collect information on all the variables considered in our application.

Information have been collected at the individual or the household level depending on the variable, but the unit of our analysis is the individual. The calculation uses required sample weights. In ECHP a person's life has been measured along the following domains: financial difficulties, basic needs and consumption, housing conditions, durables, health, social contacts and participation, and life satisfaction. The 14 non-monetary indicators ${ }^{9}$ suggested by Eurostat (2000) as best candidates to meet the requirements of 1) reflecting a negative aspect of a life pattern common to a majority of the population in the EU; 2) allowing international and intertemporal comparisons; 3) expressing a link with income poverty, are included in the analysis. These are the following:

- Financial difficulties: 1. Proportion of persons living in households that have great difficulties in making ends meet; 2 . Proportion of persons living in households that are in arrears with (re)payment of housing and/or utility bills;

- Basic necessities: 3. Proportion of persons living in households which cannot afford meat, fish or chicken every second day; 4. Proportion of persons living in households which cannot afford to buy new clothes; 5. Proportion of persons living in households which cannot afford a week's holiday away from home;

\footnotetext{
${ }^{9}$ In fact, the non-monetary indicators recommended in Eurostat (2000) are 15. We decided to drop the one belonging to the health domain, namely the proportion of people that were severely hampered in their daily activity by long-lasting health problems, since there was a considerable discontinuity between the ECHP waves for this indicator.
} 
- Housing conditions: 6. Proportion of persons living in the accommodation without a bath or shower; 7. Proportion of persons living in the dwelling with damp walls, floors, foundations, etc.; 8. Proportion of persons living in households which have a shortage of space;

- Durables: 9. Proportion of persons not having access to a car due to a lack of financial resources in the household; 10. Proportion of persons not having access to a telephone due to a lack of financial resources in the household; 11. Proportion of persons not having access to a color TV due to a lack of financial resources in the household;

- Health: 12. Proportion of persons (over 16) reporting bad or very bad health;

- Social contact: 13. Proportion of persons (over 16) who meet their friends or relatives less often than once a month (or never);

- Dissatisfaction: 14. Proportion of persons (over 16) being dissatisfied with their work or main activity.

We first calculate $E_{\delta}$ for $\delta=0,1$, and 2 separately for two sets of indicators $\mathrm{V}_{1}$ and $\mathrm{V}_{2}$, where $\mathrm{V}_{1}$ includes the indicators in the domains of financial difficulties, basic necessities, housing conditions, and durables, and $\mathrm{V}_{2}$ includes the remaining indicators. The reason for separate calculations is that for indicators covered under $V_{1}$ we have household level information, whereas for the indicators in $V_{2}$ the available information are at the individual level, with the additional constraint that the minimum age of the reportee is 16 .

We call a person socially excluded with respect to a variable in a given domain if he has been deprived of the variable for at least four years out of the six years that we observe. In addition, exclusion for a functioning occurs if the person concerned is deprived for the last three years. Thus, our calculation of the individual exclusion score explicitly takes into account the dynamic or longitudinal aspect of social exclusion. A person's exclusion in a given domain has been obtained by adding up his exclusions over the concerned variables, that is, here the deprivation score is calculated under the assumption that $w_{j}=1$ for all $j$. Since in this calculation $x_{i}$ is independent of the population size, $S U D$ holds. Calculation of non-additive measures $I_{4}$ and $I_{5}$ involving $x_{i}$ 's which are dependent on the population size is presented later in the section.

Numerical estimates of social exclusion for the EU member states are reported in Table 1. The upper part of the table presents the estimates 
for $\mathrm{V}_{1}$ while its lower part gives the analogous values for $\mathrm{V}_{2}$. The first column of the table gives the names of the countries for whom required information were available. In columns $2-4$ we present, for each country, the values of $E_{\delta}$ for $\delta=0,1$ and 2 respectively. ${ }^{10}$ The country-wise social exclusion levels are then weighted by corresponding population shares to determine the contributions of different countries to total exclusion, which are given as percentages of total exclusion in columns 5-7. From policy perspective, complete elimination of exclusion within a country would lower aggregate exclusion precisely by the percentage by which it contributes to total exclusion.

Several interesting features emerge from Table 1 . We note that the values of measures as well as percentage contributions are sensitive to the values of $\delta$. Increasingness of $E_{\delta}$ as $\delta$ increases is confirmed by values of measures shown in the table. We first analyze the upper part of the table. Portugal turns out to be the most socially excluded country. If we consider the ranking of countries from high to low exclusion, then an unambiguous sequence is Portugal, Greece, Spain, Italy, Ireland, France and Belgium. But there is no unanimous agreement about the country with minimum exclusion. The Netherlands is the country with minimum $H$, whereas $E_{1}$ and $E_{2}$ regard Denmark as the country with minimal exclusion.

As expected, the maximum percentage contribution to total exclusion comes from Portugal, whereas Denmark is the least contributing country. Belgium and the Netherlands occupy respectively the second and third position in terms of low percentage contributions. The fourth column of this part of the table shows that Portugal, Italy, Spain, and Greece, the Southern European countries, report $77.89 \%$ of social exclusion as judged by the headcount index. Their contribution to overall exclusion rises to $82.68 \%$ $(86.6 \%)$ if one uses $A\left(E_{2}\right)$. The higher contributions of these four countries is partly due to their almost average or more than average social exclusions. France and Ireland come next in the ranking by percentage contributions. A comparison between Italy and Ireland is worth noting here. Although the latter has a better position than the former with respect to $H$ and $A$, for the other measure it becomes worse off. The reason behind is that the variance of the deprivation scores is much higher in Ireland than in Italy. By percentage contributions, Ireland shows a much better picture than Italy. This is because the country has a very low population share among the member states.

\footnotetext{
${ }^{10}$ Recall that for $\delta=0$ and $1, E_{\delta}$ becomes respectively the head-count ratio, $H$, and the average deprivation score of the society, $A$.
} 
In $\mathrm{V}_{2}$ as well, Portugal is the member state with maximum exclusion and percentage contribution, Italy has the second worst off position and Ireland performs the best by showing the lowest values with respect to both the factors. Belgium, Denmark and the Netherlands also show low values for both factors. But Denmark has a better position than the other two countries by percentage contributions, and Denmark and the Netherlands perform better than Belgium by the other factor. However, the rankings among these three countries in all other situations are ambiguous. For Greece we observe relatively high values in both situations, and France and Spain, which do not have unambiguous ranking between themselves, perform worse than Greece by the either criterion. Portugal, Italy, Spain and France jointly contribute more than $83 \%$ to total exclusion by any measure. Finally, except for Portugal, the ranking of countries by any measure in $V_{2}$ is different from that in $\mathrm{V}_{1}$.

From policy point of view, the break-down of the variables into two subgroups enable us to identify the countries separately in each subset that are most susceptible to exclusion.

In Table 2 we carry out a similar analysis for Italy. The country has been divided into 11 geographic areas. ${ }^{11} \mathrm{In}_{1}$, South is the area with maximum exclusion by $E_{1}$ and $E_{2}$, while Sardegna occupies this position for $H$. Similarly, there is no unanimous agreement about the area with minimum exclusion. It is worth noting that South is only a part of the south of the country. If we add to South the remaining southern area, namely Campania, we can conclude that the southern areas contribute between $34 \%$ and $46 \%$ to total exclusion observed in Italy, depending on the measure. We note the difference with the northern regions, namely North-West, NorthEast, Lombardia and Emilia-Romagna, whose total percentage contribution ranges between $9 \%$ and $17 \%$. The other two areas with high levels of exclusion as well as percentage contributions are the two islands, Sicilia and Sardegna. In the same way in $V_{2}$, South is the geographic area with maximum exclusion, and unanimous agreement about the area with minimum exclusion is not reached. However, the northern areas occupy low exclusion positions without showing unambiguous ranking among themselves. More generally, ranking of areas by any measure is different in $V_{1}$ than that in $\mathrm{V}_{2}$.

The high contributing areas require attention from policy perspective for reduction of their contributions so that a higher living standard can be

\footnotetext{
${ }^{11}$ The information on the geoghaphic areas of the Italian households are available in ECHP at the Nuts 1 level.
} 
achieved.

In Table 3 we present results of deprivation scores using population size dependent weights. The measures that we apply are $I_{4}$, the symmetric mean exclusion of order $\nu$, and $I_{5}$, the Gini exclusion measure. Here we take into account the local dimension of the concept, i.e. people compare themselves with their reference society, and following Runciman (1966), we define the degree of deprivation inherent in not having access to an item as an increasing function of the proportion of persons in the society who have access to the item. Hence the weight attached to attribute $j, w_{j}$, reflects the percentage of the population in the country of residence of the individual that is not deprived from that specific attribute. ${ }^{12}$ The upper part of the table presents the estimates for $V_{1}$ while its lower part gives the analogous values for $\mathrm{V}_{2}$. In columns 2-4 we present, for each country, the values of $I_{4}$, for $\nu=0.5,1$ and 2 respectively. The parameter $\nu$ is the sensitivity parameter, the more positive it is, the more sensitive the index will be to the functioning failures of the more deprived. In column 5 the values of the Gini exclusion measure, $I_{5}$, are reported.

The results are strikingly different from the analysis of Table 1 in the case of both $\mathrm{V}_{1}$ and $\mathrm{V}_{2}$. South European countries split into two groups located at the opposite side of the ranking with respect to $I_{4}$. On the one hand, Portugal and Greece are still the most deprived countries, while Spain and Italy now with Denmark are the countries where social exclusion is lowest. The latter is also the country with minimum exclusion according to the Gini measure, and the position of Spain is unchanged as well, being the third in the ranking from lowest to highest exclusions. When we consider relatively high exclusions values (more than 35), starting with Greece the ranking of countries from low to high exclusion by the Gini measure is Greece, Italy, the Netherlands, Ireland and Portugal. Another notable difference with the previous unweighted case is that of the Netherlands. It is now a member state with a relatively high level of social exclusion according to all the measures.

For $\mathrm{V}_{2}$, the domains of health, social contact and dissatisfaction, the values of $I_{4}$ are quite similar among all the countries, while we observe more variance for $I_{5}$. The lowest excluded country by $I_{4}$ is always Spain, followed by Portugal when $\nu=0.5$ and 1 , and Denmark when $\nu=2$. On the contrary, Portugal is the most excluded country when disadvantage is evaluated with

\footnotetext{
${ }^{12}$ More precisely, we assume that, if the percentage of the population not deprived of functioning $j$ lies in the interval $(10(i-1), 10 i]$, where $i=1,2, \ldots, 10$, then $w_{j}=i$. If nobody is excluded from $j$, then the definition of the characteristic function ensures that deprivation with respect to $j$ is zero.
} 
the Gini measure, while France is the country with the highest level of exclusion by $I_{4}$. The Netherlands is confirmed to be a member state that occupies a low position in the scale from better to worse. The same is true also for Italy.

Naturally, the changes we observe in ranking of countries in both $V_{1}$ and $\mathrm{V}_{2}$ in comparison with the earlier case are a consequence of assignment of population size dependent weights to alternative functionings.

\section{Conclusions}

Social exclusion refers to inability of a person to participate in basic day-today economic and social activities of life.

In this paper we have developed an axiomatic approach to the measurement of social exclusion and identified the class of subgroup decomposable measures of exclusion. We have also suggested a dominance criterion for ranking two societies by symmetric additive exclusion measures under constancy of population size and total deprivation score. An application of the decomposable and non-decomposable measures considered in the paper has been made using European Union data.

Several extensions of our analysis are possible. First, a characterization of some class of measures, for example of $E_{\delta}$, will be quite interesting. Second, extension of our dominance criterion to the cases of nonadditive measures, variable total and variable population size will be worthwhile. Finally, we have considered only subgroup decomposability. We can as well consider decomposition of population exclusion by attributes and study the impact of each of them on the aggregate exclusion. This will enable us to identify the attributes that are more/less susceptible to social exclusion.

\section{References}

[1] Akerlof, G.A. (1997): "Social Distance and Social Decision", Econometrica, 65, 1005-1027.

[2] Atkinson, A.B. (1970): "On the Measurement of Inequality", Journal of Economic Theory, 2, 244-263.

[3] Atkinson, A.B. (1998): "Social Exclusion, Poverty and Unemployment", CASE/4, Centre for Analysis of Social Exclusion, London School of Economics, 1-20. 
[4] Atkinson, A.B., B. Cantillon, E. Marlier, and B. Nolan (2002): Social Indicators: The EU and Social Inclusion, Oxford.

[5] Basu, K. and J.E. Foster (1998): "On Measuring Literacy", Economic Journal, 108, 1733-1749.

[6] Bossert, W., C. D'Ambrosio and V. Peragine (2003): "Social Exclusion and Deprivation", DIW Berlin, mimeo.

[7] Bourguignon, F. and S.R. Chakravarty (2003): "The Measurement of Multidimensional Poverty", Journal of Economic Inequality, 1, 25-49.

[8] Bradshaw, J., Williams, J., Levitas, R., Pantazis, C., Patsios, D., Townsend, P., Gordon, D. and S. Middleton (2000): "The Relationship Between Poverty and Social Exclusion in Britain", Paper presented at the 26th General Conference of the International Association for Research in Income and Wealth, Cracow, Poland.

[9] Duffy, K. (1995): "Social Exclusion and Human Dignity in Europe", Council of Europe, Strasbourg.

[10] Eurostat (2000): "European Social Statistics. Income, Poverty and Social Exclusion", THEME 3, Population and social conditions, Luxembourg.

[11] Fulkerson, D.R. and H.J. Ryser (1962): "Multiplicities and Mininal Widths for (0-1) Matrices", Canadian Journal of Mathematics, 14, 498508.

[12] Klasen, S. (1998): "Social Exclusion and Children in OECD Countries: some conceptual issues", Centre for Educational Research and Innovation, OECD.

[13] Marshall, A.W. and I. Olkin (1979): Inequalities: Theory of Majorization and Its Applications, Academic Press.

[14] Mejer, L. (2000): "Statistics on Social Exclusion: the EU Methodological Approach", Eurostat, Unit E2 Living Conditions.

[15] Muirhead, R.F. (1903): "Some Methods Applicable to Identities and Inequalities of Symmetric Algebraic Functions of n Letters", Proceedings of Edimburgh Mathematical Society, 21, 144-157. 
[16] Paugam, S. and H. Russell (2000): "The Effects of Employment Precarity and Unemployment on Social Isolation", in: D. Gallie and S. Paugam (eds.), Welfare regimes and the experience of unemployment in Europe, Oxford University Press, Oxford.

[17] Room, G. (1995): Beyond the Threshold: the Measurement and Analysis of Social Exclusion, Policy Press, Bristol.

[18] Rowntree Foundation (1998): "The Report of Key Indicators of Poverty and Social Exclusion", www.parliament.the-stationeryoffice.co.uk/pa/cm199798/cmselect/777/8060303.htm.

[19] Runciman, W.G. (1966): Relative Deprivation and Social Justice, Routledge, London.

[20] Sen, A.K. (1976): "Poverty: an Ordinal Approach to Measurement", Econometrica, 44, 219-231.

[21] Sen, A.K. (1985): Commodities and Capabilities, North Holland, Amsterdam.

[22] Sen, A.K. (1998): "Social Exclusion and Economic Measurement", paper presented at the 25th General Conference of the International Association for Research in Income and Wealth, Cambridge, UK.

[23] Tsakloglou, P. and F. Papadopoulos (2001): "Identifying Population Groups at High Risk of Social Exclusion", forthcoming in: R. Muffels and P. Tsaklogou (eds.), Social exclusion in European welfare states, Edward Elgar, Cheltenham.

[24] Tsui, K-Y. (1999): "Multidimensional Inequality and Multidimensional Generalized Entropy Measures: An axiomatic derivation", Social Choice and Welfare, 16, 145-157.

[25] UK House of Commons (1999): "Poverty and Social Exclusion (national strategy) Bill", www.parliament.the-stationeryoffice.co.uk/pa/cm199899/cmbills/045/1999045.htm. 
Table 1: Social Exclusion in EU Member-States (1994-1999).

\begin{tabular}{|c|c|c|c|c|c|c|}
\hline & \multicolumn{3}{|c|}{ Values of $E_{\delta}$} & \multicolumn{3}{|c|}{ Percentage Contributions Based on: } \\
\hline & $\begin{array}{c}\mathrm{E}_{0} \\
\text { (head-count } \\
\text { ratio, } \mathrm{H} \text { ) }\end{array}$ & $\begin{array}{c}E_{1} \\
\text { (average deprivation } \\
\text { score, } A \text { ) }\end{array}$ & $E_{2}$ & $\begin{array}{c}\mathrm{E}_{0} \\
\text { (head-count } \\
\text { ratio, } \mathrm{H} \text { ) }\end{array}$ & $\begin{array}{c}\mathrm{E}_{1} \\
\text { (average deprivation } \\
\text { score, } \mathrm{A} \text { ) }\end{array}$ & $E_{2}$ \\
\hline \multicolumn{7}{|c|}{ (2) } \\
\hline Belgium & 0.208 & 0.340 & 0.873 & 2.66 & 2.00 & 1.54 \\
\hline Denmark & 0.177 & 0.239 & 0.411 & 1.88 & 1.17 & 0.60 \\
\hline Greece & 0.594 & 1.598 & 6.101 & 17.65 & 21.78 & 25.03 \\
\hline Spain & 0.510 & 0.906 & 2.222 & 19.15 & 15.61 & 11.53 \\
\hline France & 0.324 & 0.534 & 1.291 & 8.91 & 6.75 & 4.91 \\
\hline Ireland & 0.352 & 0.708 & 2.181 & 4.82 & 4.45 & 4.13 \\
\hline Italy & 0.466 & 0.813 & 2.089 & 18.77 & 15.02 & 11.62 \\
\hline Netherlands & 0.164 & 0.274 & 0.687 & 3.85 & 2.95 & 2.23 \\
\hline Portugal & 0.703 & 2.077 & 8.764 & 22.32 & 30.27 & 38.42 \\
\hline Total & 0.490 & 1.068 & 3.551 & 100 & 100 & 100 \\
\hline \multicolumn{7}{|l|}{ V2 } \\
\hline Belgium & 0.061 & 0.063 & 0.068 & 2.71 & 2.50 & 2.17 \\
\hline Denmark & 0.034 & 0.035 & 0.037 & 1.41 & 1.30 & 1.12 \\
\hline Greece & 0.074 & 0.078 & 0.087 & 8.69 & 8.14 & 7.29 \\
\hline Spain & 0.087 & 0.088 & 0.091 & 12.42 & 11.20 & 9.32 \\
\hline France & 0.082 & 0.092 & 0.115 & 10.43 & 10.45 & 10.47 \\
\hline Ireland & 0.022 & 0.023 & 0.025 & 1.21 & 1.12 & 0.96 \\
\hline Italy & 0.168 & 0.198 & 0.264 & 28.29 & 29.52 & 31.75 \\
\hline Netherlands & 0.032 & 0.034 & 0.039 & 3.05 & 2.92 & 2.72 \\
\hline Portugal & 0.254 & 0.295 & 0.381 & 31.78 & 32.85 & 34.21 \\
\hline Total & 0.122 & 0.137 & 0.170 & 100 & 100 & 100 \\
\hline
\end{tabular}

V1 considers jointly the variables included in the domains of financial difficulties, basic necessities, housing conditions, durables.

V2 considers jointly the variables included in the domains of health, social contact and dissatisfaction.

The values reported are per persons, with the additional constraint of age being at least 16 for V2. 
Table 2: Social Exclusion in Italy by Geographic Areas (1994-1999).

\begin{tabular}{|c|c|c|c|c|c|c|}
\hline & \multicolumn{3}{|c|}{ Values of $E_{\delta}$} & \multicolumn{3}{|c|}{ Percentage Contributions Based on: } \\
\hline & $\begin{array}{c}\mathrm{E}_{0} \\
\text { (head-count } \\
\text { ratio, H) }\end{array}$ & $\begin{array}{c}\mathrm{E}_{1} \\
\begin{array}{c}\text { (average deprivation } \\
\text { score, } \mathrm{A})\end{array} \\
\end{array}$ & $E_{2}$ & $\begin{array}{c}\mathrm{E}_{0} \\
\text { (head-count } \\
\text { ratio, H) }\end{array}$ & $\begin{array}{c}E_{1} \\
\text { (average deprivation } \\
\text { score, } A \text { ) }\end{array}$ & $E_{2}$ \\
\hline \multicolumn{7}{|c|}{ 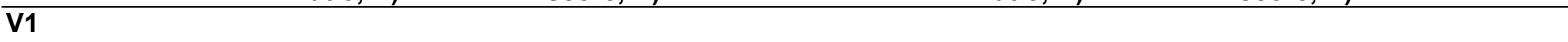 } \\
\hline North West & 0.202 & 0.314 & 0.639 & 4.06 & 3.62 & 2.86 \\
\hline Lombardia & 0.222 & 0.300 & 0.514 & 4.90 & 3.81 & 2.54 \\
\hline North East & 0.215 & 0.297 & 0.510 & 5.63 & 4.45 & 2.98 \\
\hline Emilia-Romagna & 0.225 & 0.239 & 0.277 & 2.86 & 1.74 & 0.79 \\
\hline Centre & 0.405 & 0.591 & 1.202 & 11.39 & 9.54 & 7.55 \\
\hline Lazio & 0.388 & 0.611 & 1.357 & 5.46 & 4.93 & 4.26 \\
\hline Abruzzo-Molise & 0.416 & 0.574 & 1.005 & 6.87 & 5.43 & 3.70 \\
\hline Campania & 0.542 & 1.016 & 2.878 & 12.19 & 13.09 & 14.44 \\
\hline South & 0.679 & 1.440 & 4.308 & 21.70 & 26.39 & 30.71 \\
\hline Sicilia & 0.665 & 1.243 & 4.025 & 13.49 & 14.47 & 18.23 \\
\hline Sardegna & 0.696 & 1.326 & 3.249 & 11.46 & 12.51 & 11.93 \\
\hline Total & 0.466 & 0.813 & 2.089 & 100 & 100 & 100 \\
\hline \multicolumn{7}{|l|}{ V2 } \\
\hline North West & 0.105 & 0.125 & 0.178 & 5.70 & 5.79 & 6.18 \\
\hline Lombardia & 0.090 & 0.107 & 0.150 & 5.53 & 5.66 & 5.92 \\
\hline North East & 0.098 & 0.112 & 0.143 & 7.02 & 6.84 & 6.53 \\
\hline Emilia-Romagna & 0.122 & 0.130 & 0.146 & 4.18 & 3.80 & 3.22 \\
\hline Centre & 0.164 & 0.188 & 0.241 & 12.42 & 12.15 & 11.70 \\
\hline Lazio & 0.139 & 0.166 & 0.227 & 5.47 & 5.59 & 5.72 \\
\hline Abruzzo-Molise & 0.140 & 0.167 & 0.220 & 6.24 & 6.31 & 6.25 \\
\hline Campania & 0.212 & 0.248 & 0.329 & 13.58 & 13.56 & 13.47 \\
\hline South & 0.242 & 0.293 & 0.407 & 21.42 & 22.08 & 23.00 \\
\hline Sicilia & 0.155 & 0.187 & 0.263 & 8.78 & 9.04 & 9.54 \\
\hline Sardegna & 0.204 & 0.227 & 0.280 & 9.66 & 9.17 & 8.48 \\
\hline Total & 0.168 & 0.198 & 0.264 & 100 & 100 & 100 \\
\hline
\end{tabular}

V1 considers jointly the variables included in the domains of financial difficulties, basic necessities, housing conditions, durables.

V2 considers jointly the variables included in the domains of health, social contact and dissatisfaction.

The values reported are per persons, with the additional constraint of age being at least 16 for V2. 
Table 3: Social Exclusion in EU Member-States (1994-1999).

\begin{tabular}{lcccc}
\hline & \multicolumn{3}{c}{ Values of $\mathbf{I}$} \\
\cline { 2 - 5 } & $\begin{array}{c}\mathbf{I}_{\mathbf{4}} \\
(\mathbf{v}=\mathbf{0 . 5})\end{array}$ & $\begin{array}{c}\mathbf{I}_{\mathbf{4}} \\
\mathbf{( v = 1 )}\end{array}$ & $\begin{array}{c}\mathbf{I}_{\mathbf{4}} \\
\mathbf{( v = 2 )}\end{array}$ & $\mathbf{I}_{\mathbf{5}}$ \\
\hline $\mathbf{V 1}$ & & & \\
Belgium & 13.744 & 15.208 & 19.334 & 32.641 \\
Denmark & 12.043 & 12.681 & 14.456 & 17.958 \\
Greece & 17.358 & 19.608 & 24.216 & 35.383 \\
Spain & 12.259 & 13.657 & 16.983 & 26.727 \\
France & 13.393 & 14.704 & 18.218 & 24.216 \\
Ireland & 16.118 & 18.242 & 23.170 & 57.959 \\
Italy & 11.522 & 13.082 & 17.151 & 38.593 \\
Netherlands & 15.330 & 16.717 & 20.479 & 43.162 \\
Portugal & 17.004 & 19.774 & 25.249 & 80.245 \\
& & & & \\
& & & & \\
V2 & & & & \\
Belgium & 10.293 & 10.361 & 10.568 & 13.333 \\
Denmark & 10.282 & 10.338 & 10.495 & 12.488 \\
Greece & 10.435 & 10.530 & 10.809 & 12.449 \\
Spain & 9.261 & 9.293 & 9.391 & 11.777 \\
France & 11.056 & 11.267 & 11.833 & 13.398 \\
Ireland & 10.295 & 10.353 & 10.516 & 16.781 \\
Italy & 10.471 & 10.758 & 11.526 & 18.312 \\
Netherlands & 10.623 & 10.756 & 11.136 & 14.098 \\
Portugal & 9.734 & 10.015 & 10.724 & 28.515 \\
& & & & \\
\hline
\end{tabular}

V1 considers jointly the variables included in the domains of financial difficulties, basic necessities, housing conditions, durables. V2 considers jointly the variables included in the domains of health, social contact and dissatisfaction.

The values reported are per persons, with the additional constraint of age being at least 16 for V2. 